Website: http://revistas.lamolina.edu.pe/index.php/acu/index

(C) Universidad Nacional Agraria La Molina, Lima - Perú

\title{
Impacto de heladas meteorológicas y agronómicas en alfalfa (Medicago sativa L.) en la región Puno
}

\author{
Impact meteorological and agronomic frost on alfalfa (Medicago sativa L.) in the Region Puno
}

\author{
R. A. Carbonel ${ }^{1}$; Javier Arias Carbajal2*; Percy Zorogastua ${ }^{2}$; Fernando Passoni²; Edgardo Vilcara ${ }^{2}$; Manuel Valencia ${ }^{3}$ \\ *Autor de correspondencia
}

\section{Resumen}

El objetivo de este estudio fue caracterizar las heladas meteorológicas, agronómicas y el impacto en el cultivo de alfalfa (Medicago Sativa L.) para la región Puno, mediante un análisis físico-estadístico de la información obtenida a partir de observaciones diarias de las temperaturas mínimas, máximas y precipitación pluvial provenientes de una red de 18 estaciones meteorológicas de un período de 17 años (1996/2013). El análisis de los datos de las estaciones meteorológicas agrupados con la técnica de análisis de conglomerados, produjo tres clústeres, determinándose la frecuencia anual y por período de heladas meteorológicas; la fecha promedio de ocurrencia de primera y última helada; y la duración del período libre de heladas tanto para heladas meteorológicas como agronómicas. La caracterización de las heladas indica una estrecha relación de la temperatura y rendimiento de la alfalfa, la cual está expuesta durante gran parte del año a las heladas meteorológicas y agronómicas.

Palabras clave: Alfalfa; Medicago sativa; heladas meteorológicas y agronómicas; primera helada; última helada; período libre de helada; región Puno.

\begin{abstract}
The objective of this study was to characterize the meteorological, agronomic frosts and the impact on alfalfa cultivation (Medicago sativa L.) crops at Puno region, made by a yearly collecting data and statistical analysis of information obtained from daily observations of the minimum and maximum temperatures and rainfall taken from a 18-weather stations network in 17 years (1996/2013). Results produced three clusters grouped by determining the time of weather frost, the average date of occurrence of the first and last frost and duration of frost-free period for both agronomic and freezing weather. The characterization results indicate a close linear relationship between temperature and production of biomass and dry matter of alfalfa pastures, which are exposed almost the whole year to both agronomic and frozen weather.
\end{abstract}

Keywords: Alfalfa, Medicago sativa L.; meteorological and agronomic frost; first frost; last frost; frost-free period; region Puno.

\section{Introducción}

Puno es la región de mayor riesgo climático en el Perú, en comparación con otros espacios habitados del mundo. La región sufre intermitente e irregularmente problemas de sequías e inundaciones aunque el riesgo mayor proviene de las heladas. En este fenómeno, no solo deben considerarse las caídas dramáticas de temperatura (Manarelli, 1988; Martínez et al., 2007; Matias, 2007), hasta llegar a varios grados bajo cero, sino que tiende a una muy alta variabilidad en términos de estacionalidad, por lo que es realmente difícil establecer períodos para realizar cultivos, en los cuales no ocurran heladas letales.

Las condiciones geográficas de la región, hacen que el peligro de heladas persista todo el año, impidiendo a la actividad agrícola, una característica de elevado riesgo. $\mathrm{Al}$ inicio del ciclo vegetativo "en primavera", los cultivos tienen mayor sensibilidad a las bajas temperaturas, que van en aumento conforme avanza la estación. A fines del mismo período y "en otoño" la presencia de temperaturas bajas prematuras puede afectar, total o parcialmente, al dosel de las plantas para poder obtener cosechas.

En la presente investigación se priorizó el cultivo de alfalfa, la cual fue seleccionada en función a la superficie agrícola cosechada, producción, rendimiento, importancia socioeconómica en la zona y por servir como alimento del ganado en la época de estiaje.

La alfalfa es sembrada desde los 300 hasta 3900 msnm, donde a mayor altitud la temperatura desciende. La exigencia de la alfalfa para la germinación requiere una temperatura óptima de 20 a $30{ }^{\circ} \mathrm{C}$ (Anaya, 2004). Sin embargo, el proceso de germinación se puede iniciar desde los $2^{\circ} \mathrm{C}$ hasta los $38^{\circ} \mathrm{C}$ como máximo (Del pozo, 1974).

\footnotetext{
${ }^{1}$ Minera Antapaccay, Gerencia de Relaciones Comunitarias. Email: Rolando.Carbonell@glencore.com.pe

${ }^{2}$ Departamento Académico de Fitotecnia, Facultad de Agronomía, Universidad Nacional Agraria La Molina, Apartado postal 12-056 - La Molina,

Lima, Perú..Email: ariascj@lamolina.edu.pe; percyz@lamolina.edu.pe; fpassoni@lamolina.edu.pe; eavilcara@lamolina.edu.pe

${ }_{3}^{3}$ Departamento Académico de Suelos, Facultad de Agronomía, Universidad Nacional Agraria La Molina, Apartado postal 12-056 - La Molina, Lima,

Perú. Email: mvalencia@lamolina.edu.pe
} 
En la etapa de crecimiento vegetativo, la temperatura óptima requerida es de 14 a $18^{\circ} \mathrm{C}$, con un mínimo de $5{ }^{\circ} \mathrm{C}$ y un máximo de $30^{\circ} \mathrm{C}$ (Del Pozo, 1974; Mayhua et al., 2008; D'Attellis, 2005).

Algunos autores, sostienen que la corona de alfalfa puede soportar temperaturas mínimas de hasta $10{ }^{\circ} \mathrm{C}$ bajo cero, en estado inactivo para rebrotar, cuando las condiciones de temperatura y humedad sean favorables. Para la etapa
de floración, la temperatura óptima debe fluctuar entre los 18 y $28^{\circ} \mathrm{C}$, acompañado de una temperatura nocturna de $18{ }^{\circ} \mathrm{C}$. La alfalfa requiere una precipitación promedio de 900 a $1200 \mathrm{~mm}$ al año (D'Attellis, 2005).

Los suelos deben ser de textura franca. El pH del suelo oscila entre 4 y 8,5; siendo el óptimo de 6,2 a 7,5. Se recomienda que los suelos para este cultivo, deben ser profundos con contenido de materia orgánica mayor al 4\% (Mayhua et al., 2008).

El objetivo del trabajo fue caracterizar las heladas meteorológicas y agronómicas en la Región Puno, determinando las fechas promedio de la primera y última helada, el período libre de heladas y el impacto de las mismas en el rendimiento del cultivo de alfalfa (Medicago sativa L.).

\section{Materiales y métodos}

La investigación fue no experimental, cuantitativa, de tipo descriptivo, basada en el procesamiento estadístico y análisis de información meteorológica proporcionada por el Servicio Nacional de Hidrología y Meteorología (SENAMHI). En la caracterización de las heladas meteorológicas y agronómicas, se consideró el procedimiento estándar de control de calidad de la información meteorológica. Edáficamente, se consideró las características físico-químicas principales del suelo Ilave, un Typic Ustifluvents (Soil Survey Staff, 2014) formado a partir de sedimentos finos de origen lacustre (Tabla 1).

Pentium 4 de 1,8 GHz; escáner Modelo XOL; plotter HP Design Jet. Las lecturas de la información meteorológica, fueron tomadas de las Estaciones Meteorológicas Ordinarias, localizadas en los sitios identificados en la Tabla 2. La selección de Estaciones fue realizada, tomando en cuenta la proximidad al Lago Titicaca y en los lugares donde la alfalfa fue identificada en la información agrícola. Una de ellas fue la estación de Ilave.
Tabla 1. Características Físico-Químicas del suelo Ilave (Typic Ustifluvents)

\begin{tabular}{cccccccccc}
\hline Horiz & $\begin{array}{c}\text { Profundidad } \\
(\mathrm{cm})\end{array}$ & \multicolumn{1}{c}{ Aranulometría (\%) } & $\begin{array}{c}\text { Clase } \\
\text { Arena }\end{array}$ & Arcilla & $\begin{array}{c}\text { M.O. } \% \\
\text { textural }\end{array}$ & $\begin{array}{c}\mathrm{p} \\
\text { disponible }\end{array}$ & $\begin{array}{c}\mathrm{K} \\
\mathrm{ppm}\end{array}$ & $\mathrm{ppm}$ \\
\hline Ap & $0-18$ & 50 & 46 & 4 & F. A. & 6,6 & 1,40 & 25,4 & 179 \\
C & $18-40$ & 52 & 32 & 12 & F. A. & 6,9 & 1,20 & 31,4 & 165 \\
C2 & $40-56$ & 54 & 33 & 12 & F. A. & 6,0 & 0,40 & 30,2 & 143 \\
2C3 & $56-130$ & 26 & 48 & 26 & F. & 5,6 & 0,36 & 39,8 & 124 \\
\hline FA: Franco Arenoso & F: Franco & & & & &
\end{tabular}

En el desarrollo de labores de campo y gabinete, los equipos informáticos utilizados fueron una Computadora.

La recopilación de la información estadística del cultivo de alfalfa (Collantes, 2007) estuvo referida a las variables de producción, rendimiento y superficie cosechada provenientes de la Dirección Regional Agraria (DRA) de Puno, correspondiente a las campañas agrícolas de 1996/1997 a 2012/2013. El nivel de detalle espacial y escala temporal de las variables utilizadas en esta investigación fue distrital y anual.

En el procesamiento de la información se utilizaron los programas informáticos: ARC GIS 9,3 (Environmental Systems Research Institute, Inc.); Instat Universidad de Reading; Consulta Gráfica Estadística y Minitab versión 16 (libre disponibilidad temporal).

Tabla 2. Ubicación política y referenciada de las estaciones meteorológicas para la toma de datos climáticos

\begin{tabular}{|c|c|c|c|c|c|c|}
\hline Estación & Provincia & Distrito & $\mathrm{X} 18$ & Y18 & $\begin{array}{l}\text { Altitud } \\
(\mathrm{msnm})\end{array}$ & $\begin{array}{l}\text { Zona de } \\
\text { vida }\end{array}$ \\
\hline Arapa & Azángaro & Arapa & $1,025,006,213$ & $8,320,830,695$ & 3970 & P obhM \\
\hline Ayaviri & Melgar & Ayaviri & $974,478,877$ & $8,350,130,471$ & 3938 & P o bhM \\
\hline Azángaro & Azángaro & Azángaro & $1,017,739,122$ & $8,345,517,772$ & 3953 & P o bhM \\
\hline Cabanillas & Lampa & Cabanillas & $999,251,811$ & $8,265,488,183$ & 3993 & P o bhM \\
\hline Capachica & Puno & Capachica & $1,053,301,185$ & $8,266,847,674$ & 4002 & P o bhM \\
\hline Cojata & Huancané & Cojata & $1,106,692,630$ & $8,331,077,400$ & 4380 & bhM \\
\hline Desaguadero & Chucuito & Desaguadero & $1,136,760,187$ & $8,158,677,248$ & 3860 & P o bhM \\
\hline Huancané & Huancané & Huancané & $1,063,216,081$ & $8,312,420,137$ & 3986 & P o bhM \\
\hline Huaraya Moho & Moho & Moho & $1,091,919,550$ & $8,291,000,220$ & 3961 & P o bhM \\
\hline Ilave & El Collao & Ilave & $1,073,558,702$ & $8,214,249,725$ & 3986 & P o bhM \\
\hline Juli & Chucuito & Juli & $1,093,214,038$ & $8,200,548,227$ & 4393 & bhM \\
\hline Juliaca & San Román & Juliaca & $1,020,153,499$ & $8,283,933,594$ & 4000 & P o bhM \\
\hline Lampa & Lampa & Lampa & $9,970,963,779$ & $8,296,993,077$ & 3949 & P o bhM \\
\hline Pucara & Lampa & Pucara & $9,978,545,886$ & $8,332,733,791$ & 3980 & ph-SAT \\
\hline Puno & Puno & Puno & $1,034,074,652$ & $8,244,256,069$ & 3999 & ph-SAT \\
\hline Santa Rosa & El Collao & Santa Rosa & $9,530,505,376$ & $8,378,733,119$ & 3999 & tp-AT \\
\hline Taraco & Huancané & Taraco & $1,039,243,920$ & $8,301,695,239$ & 3999 & $\mathrm{bhM}$ \\
\hline Yunguyo & Yunguyo & Yunguyo & $1,134,717,871$ & $8,188,077,480$ & 3965 & bhM \\
\hline
\end{tabular}

La caracterización climática fue realizada utilizando la serie histórica, período 1996 a 2013, de datos diarios de precipitación $(\mathrm{mm})$, temperaturas mínimas $\left({ }^{\circ} \mathrm{C}\right) \mathrm{y}$ máximas $\left({ }^{\circ} \mathrm{C}\right)$ de la Red de 18 Estaciones Meteorológicas convencionales del SENAMHI, obtenidos de la lectura directa de los termómetros de mínima, termómetro de máxima y pluviómetro instalados dentro del abrigo Meteorológico, según las normas de la Organización Meteorológica Mundial (OMM, 1996). 
Para la zona de Ilave, los registros climáticos marcaron los valores que se consignan en la Tabla 3. Se indica la temperatura media y la precipitación, así como los regímenes, tanto de humedad, como de temperatura, bajo los cuales se desarrollaron los suelos.

Tabla 3. Valores de temperatura media y precipitación en la zona de Ilave (años 2002-2013)

\begin{tabular}{lcc}
\hline \multicolumn{1}{c}{ Período } & Temperatura media $\left({ }^{\circ} \mathrm{C}\right)$ & Precipitación $(\mathrm{mm})$ \\
\hline Enero & 10,1 & 153,8 \\
Febrero & 10,1 & 109,7 \\
Marzo & 10,0 & 127,2 \\
Abril & 9,0 & 40,7 \\
Mayo & 16,8 & 11,5 \\
Junio & 5,7 & 4,7 \\
Julio & 6,7 & 20,7 \\
Agosto & 5,7 & 13,4 \\
Septiembre & 6,5 & 22,2 \\
Octubre & 7,8 & 34,6 \\
Noviembre & 9,3 & 47,1 \\
Diciembre & 10,4 & 88,6 \\
Promedio anual & 8,5 & $*$ \\
Total anual & $*$ & 674,2 \\
Régimen & Isomésico & Ustico \\
\hline
\end{tabular}

\section{Control de calidad de la información meteorológica}

El control de calidad de la información de las variables temperatura mínima, temperatura máxima y precipitación, fue realizada mediante el software Consulta Gráfica Estadística, elaborado por la Oficina General de Estadística e Informática del SENAMHI. Las principales fuentes de error observadas durante el uso del software, fueron datos duplicados, cambios de instrumental y reubicación de estaciones. Por ello, para disminuir los errores de la fuente original de datos, se aplicó el criterio de rangos en la cual la variable en estudio no tenía que ser mayor ni menor entre ciertos límites razonables. Para el procesamiento de las fechas de primera, última y período libre de heladas, fue utilizado el software INSTAT, de la Universidad de Reading, Inglaterra, versión 3,36 (2005), el cual dio la información en días julianos.

\section{Elaboración de mapas}

En la elaboración de los mapas de caracterización de heladas agronómica y meteorológica para la región Puno, se utilizó el software ArcGIS 9,3; (Environmental Systems Research Institute, Inc.), que es una herramienta del SIG (Sistema de Información Geográfica) de uso bastante extendido por sus avanzadas capacidades de visualización, consulta y análisis de información geográfica, además de las numerosas herramientas de integración de datos desde diversos tipos de fuentes y herramientas de edición.

Para la elaboración de los mapas de frecuencia anual de heladas meteorológicas, se consideraron como variables las fechas promedio de ocurrencia de la primera y última helada y el período medio libre de heladas. Para graficar el mapa, o los datos fueron transformados del formato vectorial a raster. Para la interpolación de las variables (puntos incluidos), se usó el método de interpolación de la distancia inversa (IDW), utilizando el programa ArcGIS 9,3. Se utilizó este método de interpolación, debido a que refleja mejor la relación que hay entre la altura y la temperatura $y$, porque presupone que la variable que se representa cartográficamente, disminuye su influencia con la mayor distancia desde la ubicación de la muestra. El resultado fue la creación de archivos raster (grid).

\section{Determinación de la fecha promedio de primera helada y última helada}

La fecha promedio de la primera y última helada, correspondió a la distribución de las fechas promedios a lo largo del período en estudio considerado. Para esto, se utilizó el calendario Juliano, en el cual los días del año fueron numerados de uno a 365 , y si eran bisiestos del uno al 366; luego, se calculó el promedio de la primera y última helada, solamente con los años que registraban heladas (SENAMHI, 2010).Toda la información fue procesada con ayuda del programa estadístico Instat $+\mathrm{V} 3,36$; considerando las heladas meteorológicas $\left(0^{\circ} \mathrm{C}\right)$ y agronómicas $\left(5^{\circ} \mathrm{C}\right)$, produciendo las fechas de ocurrencia. Respecto a este último valor, se consideró como temperatura base $5^{\circ} \mathrm{C}$ para el cultivo de alfalfa.

La ocurrencia de la primera o última helada no coincidió año a año y tampoco sucedió en la fecha calculada, por eso se calculó la desviación estándar, teniendo como resultado un rango estimado en el cual podía producirse la helada.

\section{Determinación del periodo medio libre de heladas}

El período promedio libre de heladas fue considerado como aquel que comenzó el día posterior a la última helada del año anterior y finalizó con el día anterior a la primera helada del año siguiente (SENAMHI, 2010). Esto fue calculado como:

$$
\mathrm{PLH}=(365-\mathrm{UHt})+\mathrm{PHt}+1-1
$$

donde:

$\mathrm{PHL}=$ Período libre de heladas

$\mathrm{UHt}=$ Fecha de ocurrencia (en día juliano) de la última helada ocurrida en el año $t$

$\mathrm{PH}+\mathrm{t}=\mathrm{Fecha}$ de ocurrencia de la primera helada en el año $\mathrm{t}+1$

\section{Recopilación y procesamiento de información meteorológica}

En la elaboración de los mapas de caracterización de heladas agronómica y meteorológica para la región Puno, se utilizó el software ArcGIS 9,3; que es una herramienta SIG (Sistema de Información Geográfica) de uso extendido, por sus capacidades de visualización, consulta y análisis de información geográfica. 


\section{Recopilación y procesamiento de información agrícola}

La información estadística sobre la producción, rendimiento y superficie cosechada del cultivo de alfalfa, en Puno, corresponde a las campañas agrícolas de los períodos 1996/1997a 2012/2013. El nivel de detalle fue distrital y anual para la variable de producción, rendimiento y superficie cosechada. La información fue procesada utilizando la estadística descriptiva, con la cual se pudo organizar e interpretar la información procedente de las tres variables y mediante el uso de percentiles se pudo dar niveles de clasificación.

El aspecto agrícola está muy relacionado a las características de los suelos. El suelo Ilave mencionado se ha desarrollado sobre materiales de origen fluvio-lacustre y su evolución ha estado condicionada por las propiedades físicas, principalmente la textura franco arenosa $y$ las propiedades químicas, $\mathrm{pH}$ de 6,0 a 6,9 y una alta saturación de bases. El pH de este suelo no solo permite la disponibilidad de la mayoría de los nutrientes esenciales, sino que también estimula la fijación simbiótica del nitrógeno. Las bacterias del género Rhizobium no pueden desarrollarse en medios ácidos (Gómez, 2005).

La disponibilidad del fósforo en el suelo, está altamente influenciada por el $\mathrm{pH}$, siendo más favorables los suelos con $\mathrm{pH}$ neutro. Los valores de fósforo disponible, en los primeros $40 \mathrm{~cm}$ del suelo, son altos de 25,4 a 31,4 ppm (Tabla 1), lo que favorece los procesos de transformación de elementos químicos, como la mineralización, la inmovilización y la solubilizarían de fosfatos, incrementando el desarrollo de las plantas y de las poblaciones microbianas, además de contribuir al desarrollo de una estructura estable en el suelo (Alexander, 1994).

\section{Procesamiento estadístico}

Para controlar varias variables simultáneamente, la herramienta más usada fue la carta de control multivariada (Dallas,1998) propuesta por Hotelling basada en el estadístico T2 de Hotelling. Es así que la prueba de T2 de Hotelling, en el programa SPSS Statistic 17,0; en el entorno Windows, con $95 \%$ de confianza, fue utilizada para evaluar las diferencias y así determinar que estaciones meteorológicas presentan información que se encontraban por debajo o por encima del promedio general de las variables en estudio (altitud, precipitación y temperatura).

El Análisis de Conglomerados (Clústeres) fue analizado con el programa Minitab16, a fin de realizar un agrupamiento de estaciones meteorológicas según similitudes tanto en precipitación, altitud y rango de temperatura. Se formaron grupos homogéneos dentro de cada grupo y heterogéneos entre ellos. Para esta técnica de agrupamiento, se utilizó la distancia euclidiana.

El coeficiente de correlación de Pearson, fue utilizado para determinar la existencia de correlación lineal entre las variables meteorológicas y el rendimiento del cultivo de alfalfa (Domenech, 1985).
Los modelos de regresión lineal, fueron usados para determinar cuál variable o variables meteorológicas explicaban parte de la varianza del rendimiento agrícola de los cultivos. Los modelos usados, han sido estimados comprobando la normalidad del error, los valores de los parámetros y la significación. Las regresiones, fueron calculadas sobre los residuos estandarizados al cuadrado, rechazando en todos los casos, la hipótesis nula de auto correlación (Etxeberria, 1999). La ausencia de homocedasticidad, fue comprobada mediante el test de White, que en todos los casos, también se comportó satisfactoriamente.

\section{Resultados y discusión}

\section{Agrupamiento de estaciones meteorológicas}

La actividad agrícola en la región Puno se desarrolla entre los meses de septiembre a abril. Por ello, durante el período 1996-2013, con el análisis de agrupamiento, se determinaron tres grupos o clústeres de estaciones meteorológicas, según el comportamiento de las variables meteorológicas en estudio. Para esta técnica de agrupamiento de las estaciones meteorológicas, se implementaron los métodos de clasificación: jerárquico y de repartición (Figura 1).

Mediante la técnica de Análisis de Conglomerados o de Clúster, se realizó un agrupamiento de estaciones meteorológicas según la similitud de las variables en estudio, las cuales fueron precipitación, altitud y rango de temperatura, de este modo se obtuvieron grupos homogéneos, dentro de cada grupo y heterogéneos entre ellos. Para esta técnica de agrupamiento, se utilizó la distancia euclidiana.

Se determinaron tres grupos o clústeres de estaciones meteorológicas para la región Puno (Tabla 4), según el comportamiento de las variables en estudio, durante los meses de setiembre hasta abril (meses en los que se desarrolla la actividad agrícola en la región) del período 1996/2013. Para esta técnica de agrupamiento de las estaciones meteorológicas, se implementaron los métodos de clasificación: jerárquico y de repartición (K-medias).

Tabla 4. Clústeres o grupos de estaciones meteorológicas en la región Puno

\begin{tabular}{ll}
\hline Clúster & Estaciones meteorológicas \\
\hline 1 & $\begin{array}{l}\text { Huancané, Yunguyo, Desaguadero Azángaro, Ilave, } \\
\text { Puno, Cabanillas, Huaraya, Moho, Pucara, Juliaca, }\end{array}$ \\
2 & $\begin{array}{l}\text { Taraco, Capachica y Lampa } \\
\text { Arapa, Ayaviri y Santa Rosa }\end{array}$ \\
3 & Cojata \\
\hline
\end{tabular}

A partir del dendrograma de la Figura 1, fue posible obtener los siguientes clúster o grupos de estaciones meteorológicas: 


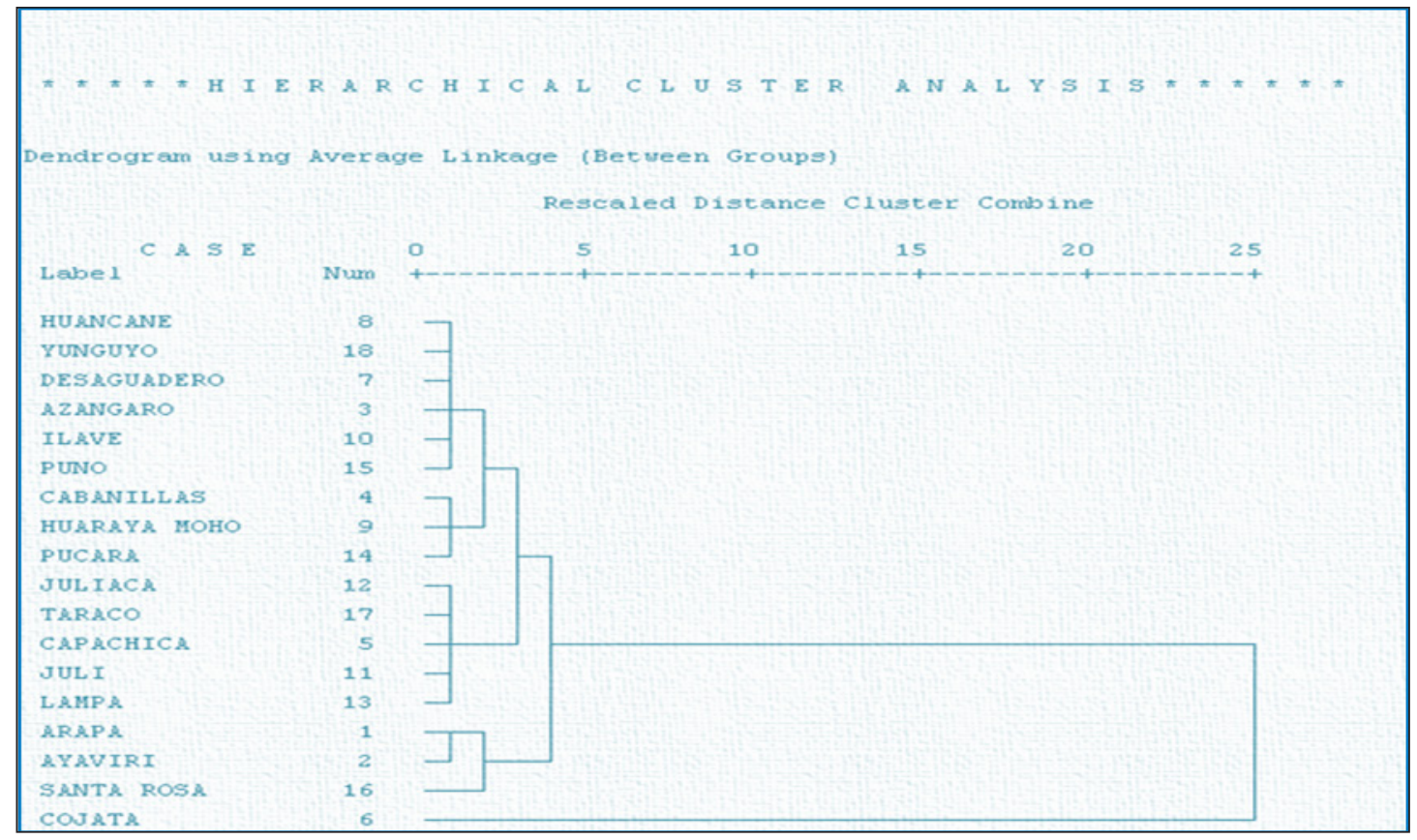

Figura 1. Dendrograma del análisis de cluster para las estaciones meteorológicas de la región Puno - Distancias Euclidianas

Tabla 5. Fechas medias de primera helada, última helada y período libre de heladas

\begin{tabular}{|c|c|c|c|c|c|c|c|c|c|c|c|}
\hline \multirow{2}{*}{ Estaciones } & \multirow{2}{*}{$\mathrm{T}^{\circ}$} & \multicolumn{3}{|c|}{ Primeras Heladas } & \multicolumn{3}{|c|}{ Ultimas Heladas } & \multicolumn{4}{|c|}{ Período Libre de Heladas } \\
\hline & & FM & IM & DS & FM & IM & DS & FM & DS & MX & $\mathrm{MN}$ \\
\hline \multirow[t]{2}{*}{ Arapa } & 0 & 92 & $-0,7$ & 1,1 & 318 & $-0,4$ & 0,8 & 139 & 57,1 & 224 & 33 \\
\hline & 5 & 15 & 4,3 & 0,6 & 350 & 4,3 & 0,8 & 30 & 6,0 & 29 & 1 \\
\hline \multirow[t]{2}{*}{ Ayaviri } & 0 & 49 & $-0,4$ & 0,5 & 344 & $-0,8$ & 1,0 & 69 & 54,6 & 181 & 8 \\
\hline & 5 & 10 & 3,1 & 1,0 & 348 & 3,4 & 1,2 & 27 & 2,7 & 13 & 1 \\
\hline \multirow{2}{*}{ Azángaro } & 0 & 102 & $-0,7$ & 0,8 & 310 & $-1,0$ & 1,5 & 157 & 38,4 & 237 & 98 \\
\hline & 5 & 10 & 4,3 & 0,7 & 347 & 3,9 & 1,2 & 28 & 28,3 & 127 & 1 \\
\hline \multirow[t]{2}{*}{ Cabanillas } & 0 & 109 & $-0,9$ & 0,9 & 289 & $-0,8$ & 0,8 & 185 & 32,6 & 248 & 117 \\
\hline & 5 & 15 & 4,0 & 1,2 & 350 & 3,9 & 0,9 & 30 & 8,1 & 37 & 1 \\
\hline \multirow[t]{2}{*}{ Capachica } & 0 & 94 & $-0,6$ & 0,7 & 309 & $-0,7$ & 0,9 & 150 & 51,6 & 232 & 51 \\
\hline & 5 & 15 & 3,9 & 0,7 & 355 & 3,9 & 0,7 & 25 & 5,1 & 17 & 1 \\
\hline \multirow[t]{2}{*}{ Cojata } & 0 & 28 & -2 & 2,7 & 358 & $-1,3$ & 1,4 & 35 & 17,9 & 58 & 1 \\
\hline & 5 & 6 & $-0,2$ & 3,6 & 30 & 1,1 & 1,7 & 11 & 0,5 & 2 & 1 \\
\hline \multirow[t]{2}{*}{ Desaguadero } & 0 & 63 & $-0,6$ & 0,8 & 331 & $-1,2$ & 1,3 & 97 & 48,7 & 185 & 21 \\
\hline & 5 & 10 & 4,0 & 1,1 & 355 & 3,8 & 1,5 & 20 & 4,5 & 19 & 1 \\
\hline \multirow[t]{2}{*}{ Huancané } & 0 & 91 & $-1,2$ & 0,6 & 317 & $-1,1$ & 0,8 & 139 & 41,2 & 227 & 38 \\
\hline & 5 & 12 & 4,0 & 0,7 & 348 & 3,8 & 1,2 & 29 & 2,2 & 10 & 1 \\
\hline \multirow[t]{2}{*}{ HurayaMoh } & 0 & 105 & $-0,6$ & 0,5 & 292 & $-0,5$ & 0,8 & 178 & 36,2 & 235 & 105 \\
\hline & 5 & 15 & 4,2 & 0,7 & 350 & 4,3 & 0,6 & 30 & 3,2 & 13 & 1 \\
\hline \multirow{2}{*}{ Ilave } & 0 & 102 & $-1,0$ & 1,0 & 314 & -1 & 1,3 & 153 & 44,9 & 214 & 64 \\
\hline & 5 & 16 & 4,2 & 0,7 & 349 & 3,7 & 1,1 & 32 & 26,3 & 119 & 1 \\
\hline \multirow[t]{2}{*}{ Juli } & 0 & 110 & $-0,9$ & 0,9 & 293 & $-0,9$ & 0,9 & 181 & 46,5 & 236 & 46 \\
\hline & 5 & 25 & 4,4 & 0,5 & 347 & 4,1 & 0,7 & 43 & 3,4 & 12 & 1 \\
\hline \multirow[t]{2}{*}{ Juliaca } & 0 & 85 & $-0,3$ & 0,2 & 325 & $-1,7$ & 2,9 & 125 & 72,3 & 243 & 42 \\
\hline & 5 & 19 & 2,7 & 1,5 & 343 & 1,4 & 4,3 & 41 & 98,6 & 243 & 1 \\
\hline \multirow[t]{2}{*}{ Lampa } & 0 & 59 & $-0,9$ & 1,0 & 346 & $-1,2$ & 1,1 & 78 & 42,0 & 150 & 17 \\
\hline & 5 & 10 & 4,0 & 0,9 & 350 & 3,6 & 1,2 & 25 & 2,9 & 14 & 1 \\
\hline \multirow[t]{2}{*}{ Pucara } & 0 & 50 & $-1,2$ & 1,2 & 335 & $-1,4$ & 1,3 & 79 & 41,3 & 153 & 3 \\
\hline & 5 & 9 & 3,3 & 2,3 & 351 & 2,7 & 2,1 & 23 & 14,7 & 61 & 1 \\
\hline \multirow{2}{*}{ Puno } & 0 & 119 & $-0,5$ & 0,4 & 285 & $-0,6$ & 0,7 & 200 & 39,1 & 246 & 122 \\
\hline & 5 & 12 & 4,4 & 0,6 & 351 & 4,4 & 0,7 & 26 & 9,7 & 41 & 1 \\
\hline \multirow[t]{2}{*}{ Santa Rosa } & 0 & 79 & $-1,3$ & 0,7 & 273 & $-1,1$ & 0,6 & 171 & 63,2 & 223 & 15 \\
\hline & 5 & 26 & 1,9 & 2,4 & 335 & 2,5 & 2,6 & 56 & 81,6 & 200 & 1 \\
\hline \multirow{2}{*}{ Taraco } & 0 & 64 & $-3,1$ & 1,6 & 323 & $-2,6$ & 1,2 & 106 & 51,9 & 197 & 17 \\
\hline & 5 & 15 & 4,3 & 0,7 & 350 & 4,4 & 0,6 & 30 & 15,0 & 66 & 1 \\
\hline \multirow{2}{*}{ Yunguyo } & 0 & 59 & $-0,8$ & 0,7 & 317 & $-0,7$ & 0,8 & 107 & 60,8 & 228 & 24 \\
\hline & 5 & 10 & 4,0 & 1,0 & 345 & 3,8 & 1,0 & 30 & 11,8 & 56 & 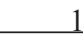 \\
\hline
\end{tabular}

$\mathrm{T}^{\circ}=$ Temperatura; $\mathrm{FM}=$ Fecha media; $\mathrm{IM}=$ Intensidad media; $\mathrm{DS}=$ Desviación estándar; $\mathrm{MX}=$ Máxima; $\mathrm{MN}=$ Mínima 


\section{Caracterización de heladas}

Debido a la importancia de la temperatura en el crecimiento y desarrollo de los cultivos, se elaboró una caracterización del régimen de heladas meteorológicas, como agronómicas (Alarcón, 1991), Para esto, se tomó como base la temperatura mínima, la cual es considerada como el elemento de mayor incidencia en el crecimiento y desarrollo de los cultivos en la región, la misma que está asociada principalmente a la altitud y a las condiciones fisiográficas (Lasso, 1997). En la Tabla 5, se presentan las fechas medias de primera helada, última helada y período medio libre de heladas meteorológica y agronómica, con la respectiva desviación estándar, así como también, la intensidad media de la helada. Las fechas promedio de primera, última y período libre de helada meteorológica se muestran en la Figura 2, pudiendo observarse que las primeras heladas meteorológicas generalmente ocurren al inicio del otoño. año en promedio, como es el caso de las estaciones que conforman el Clúster 2. En las zonas circundantes al lago, donde se ubican las estaciones pertenecientes al Clúster 1, la frecuencia disminuye de manera significativa limitando la presencia de este evento solo a los meses de invierno, esto debido al efecto termorregulador que ejerce el mismo siendo así la zona de mayor concentración de la producción agrícola en la región. Por otro lado, en la frecuencia de heladas meteorológicas para el período setiembre-abril se desarrolla la actividad agrícola en la región y en el período diciembre-febrero ocurre la etapa fenológica más crítica. La frecuencia de heladas aumenta con la altura, no siendo así en las zonas circundantes al Lago debido al efecto termorregulador que ejerce este. El período agrícola en la región coincide con la época de menor frecuencia de heladas para poder reducir el impacto de las mismas obteniendo mejores cosechas.

Las fechas promedio de primera, última y período libre

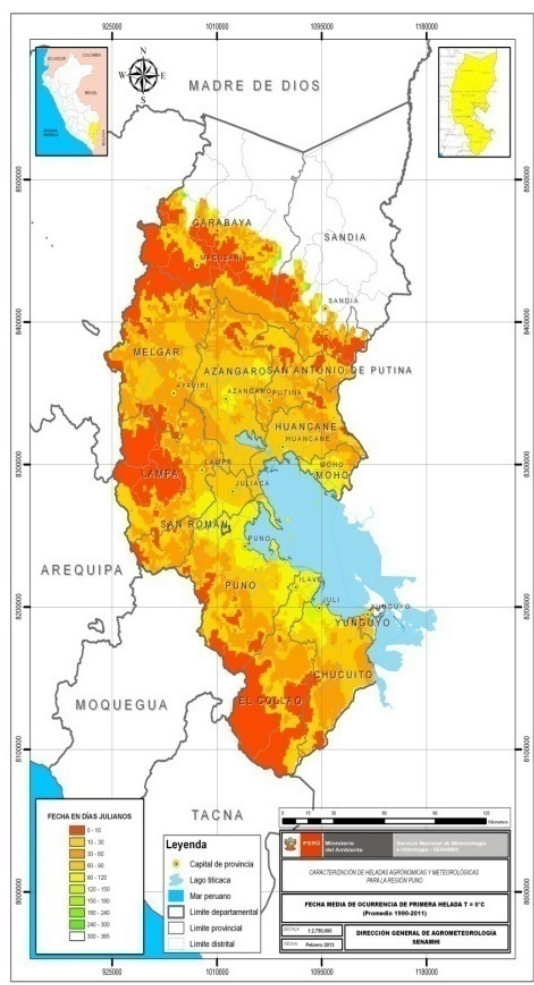

Fecha promedio de ocurrencia de primera helada meteorológica

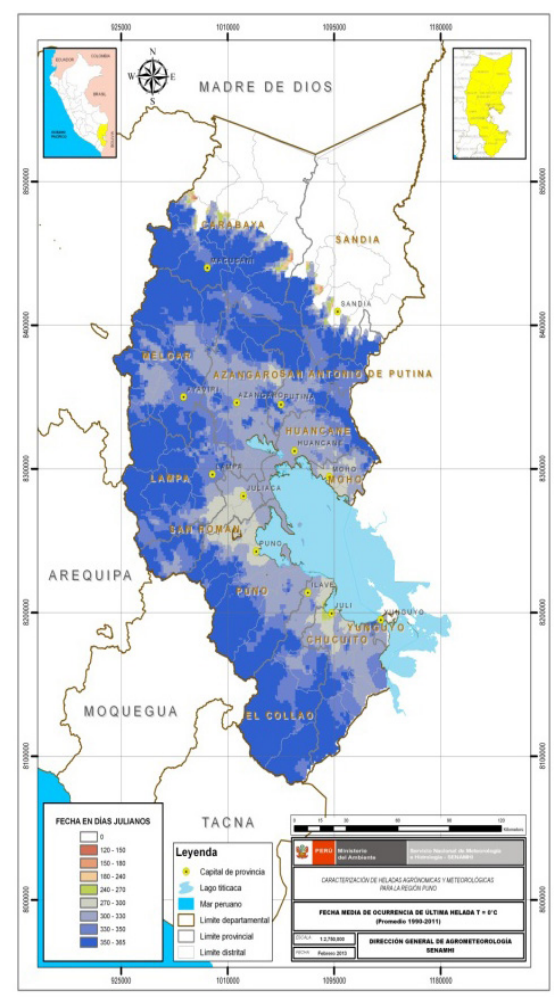

Fecha promedio de ocurrencia de última helada meteorológica

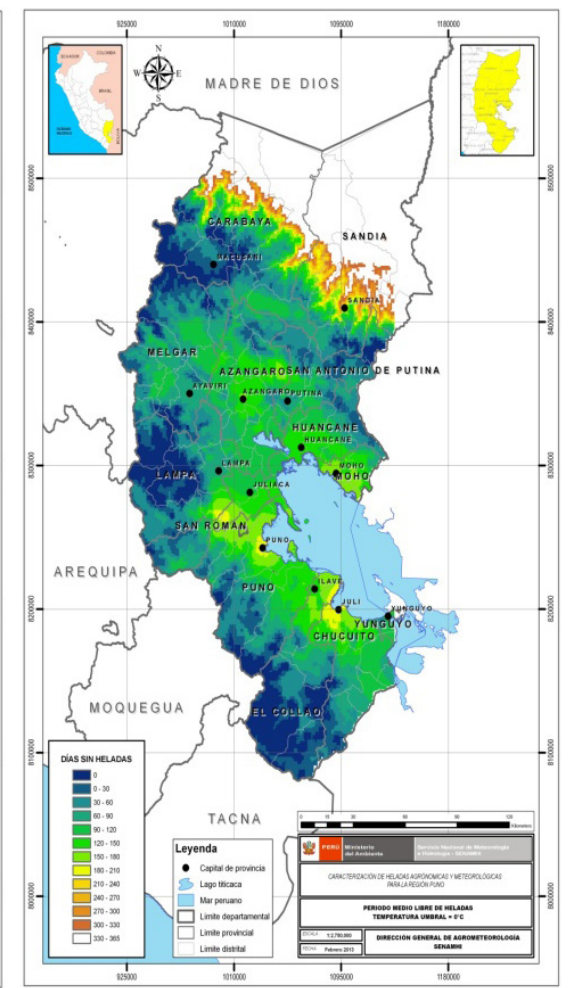

Período promedio libre de heladas meteorológicas

Figura 2. Fecha promedio de primera, última y período libre de helada meteorológica

Fuente: SENAMHI (2012).

En la frecuencia anual de heladas, la mayor frecuencia de heladas meteorológicas se presenta en las partes más alejadas al Lago Titicaca o más elevadas de la región como sucede con la estación perteneciente al Clúster 3 debido a que la gradiente de temperatura es muy marcada en zonas con mayor pendiente, pudiendo llegar, en algunas partes, a tener los 365 días del año con heladas. Mientras más se acerca al lago, la frecuencia de heladas disminuye oscilando en algunas partes a solo 150 días al de helada agronómica para el cultivo de Alfalfa (Medicago sativa) se muestran en la Figura 3. Las primeras heladas agronómicas generalmente ocurren dentro de los primeros 25 días del mes de enero; las últimas heladas ocurren a finales de la estación de primavera y el período medio libre de helada agronómica tiene una mayor duración, en las zonas cercanas al lago, alrededor de 30 días en promedio, donde también las intensidades de heladas son menores. Las estaciones pertenecientes al Clúster 1 son las que registran un mayor período libre de helada agronómica. 


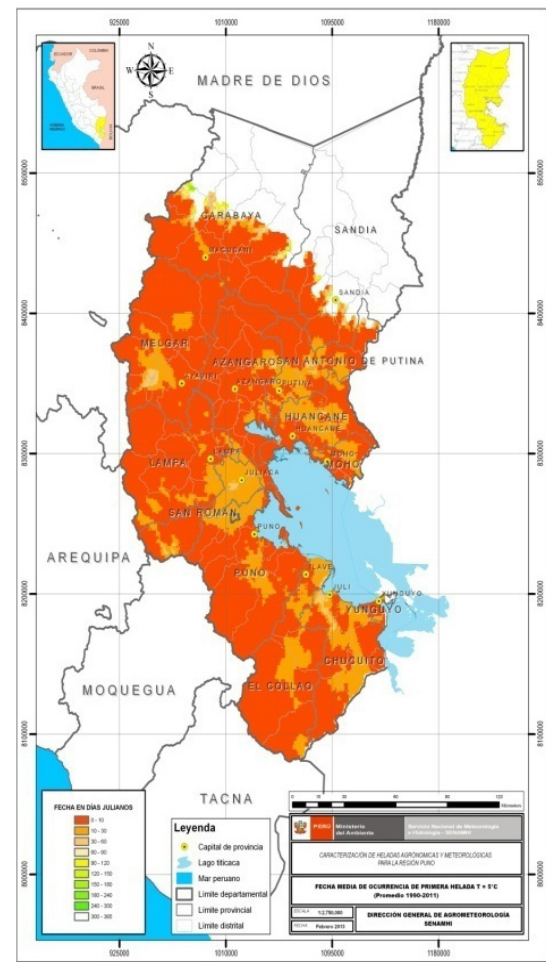

Fecha promedio de ocurrencia de primera helada agronómica $\left(\mathrm{T} \leq 5^{\circ} \mathrm{C}\right)$ para el cultivo de alfalfa

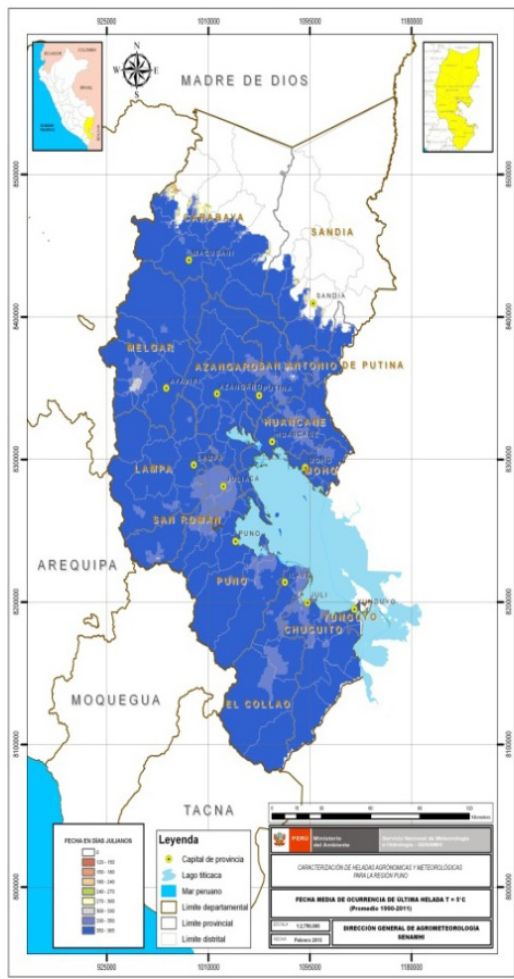

Fecha promedio de ocurrencia de última helada agronómica $\left(\mathrm{T} \leq 5^{\circ} \mathrm{C}\right)$ para el cultivo de alfalfa

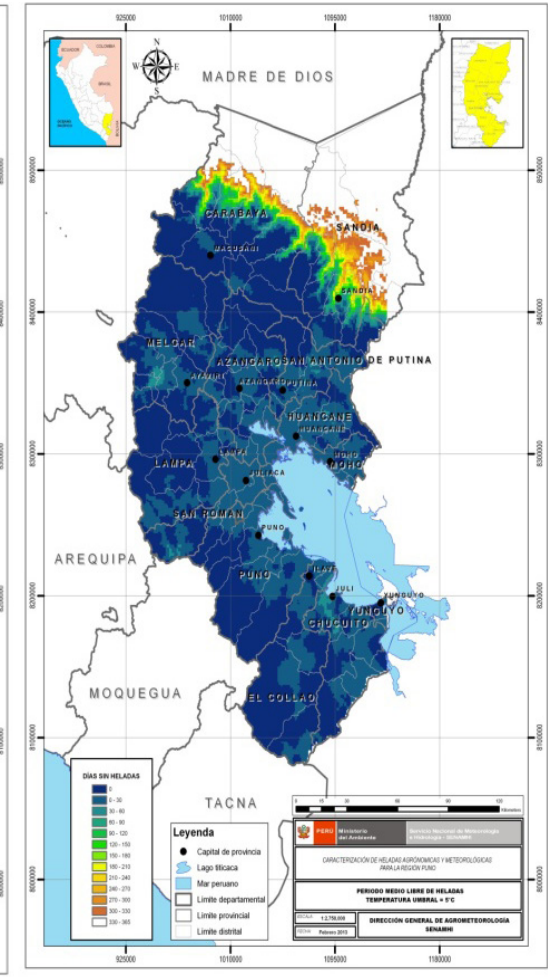

Período promedio libre de helada agronómica $\left(\mathrm{T} \leq 5^{\circ} \mathrm{C}\right)$ para el cultivo de alfalfa

Figura 3. Fecha promedio de primera, última y período libre de helada agronómica $\left(\mathrm{T} \leq 5^{\circ} \mathrm{C}\right)$ para el cultivo de alfalfa Fuente: SENAMHI (2012).

\section{Impacto de las variables climáticas en los cultivos}

El rendimiento agrícola, experimenta importantes cambios de una campaña a otra. El clima influyó en dichos cambios de forma distinta en la región, según el cultivo, las condiciones geográficas y la intensidad de las anomalías climáticas presentadas (Defina, 1945; De Fina y Ravello, 1979). Para determinar el impacto de las variables climáticas puestas en estudio en el rendimiento de los cultivos, se usaron modelos de regresión lineal general. Con este modelo de lineal general, se pretendió determinar la variable climática que explicara mejor la varianza de los rendimientos agrícolas a través del análisis estadístico.

De la relación entre el rendimiento de alfalfa (Medicago sativa) y las variables precipitación y temperatura, según el clúster asociado y el modelo generado se obtuvieron las siguientes ecuaciones de regresión lineal:

G1 Rendimiento $=19,64-$ 0,3569 Precipitación + 0,8382 Temperatura

G2 Rendimiento $=19,30-$ 0,3569 Precipitación + 0,8382 Temperatura

Estas ecuaciones, demuestran que si la temperatura promedio anual aumenta en $1{ }^{\circ} \mathrm{C}$, los rendimientos por hectárea de dicho cultivo aumentarían en $838,2 \mathrm{Kg}$ en promedio.

El análisis realizado, demostró la existencia de tres grupos de patrones térmicos espaciales con un comportamiento homogéneo tanto espacial como temporal en cada de uno de ellos. De esta manera, se determinaron tres grupos o clústeres de estaciones meteorológicas bien diferenciados en cuanto al comportamiento de las temperaturas mínimas para la región de Puno.

La zona que abarcó el Clúster 1, fue donde hubo mayor concentración de la actividad agrícola de la región, pudiendo encontrarse los más altos rendimientos en la alfalfa, esto debido a que posee un menor rango de temperatura [11,5 $\left.{ }^{\circ} \mathrm{C}-5,5^{\circ} \mathrm{C}\right]$ y una mayor temperatura media $\left(\operatorname{Tm}=11^{\circ} \mathrm{C}\right)$; la frecuencia de heladas meteorológicas, fue menor sobre todo durante el período en que los cultivos atravesaron la etapa fenológica más crítica y con un período libre de heladas agronómicas de 30 días para la alfalfa.

La zona que abarcó el Clúster 2 presentó un mayor rango de temperatura $\left[13{ }^{\circ} \mathrm{C}-6^{\circ} \mathrm{C}\right]$ así como también, una menor temperatura media $\left(\mathrm{Tm}=10^{\circ} \mathrm{C}\right)$ y con una mayor variabilidad, registrando una mayor frecuencia de heladas anual y por período, donde el período libre de heladas agronómicas tuvo una duración de 25 días para la alfalfa, lo cual fue traducido en un mayor impacto negativo.

La zona que abarcó el Clúster 3 presentó una temperatura mínima media por debajo del promedio $\left(\operatorname{Tm}=9^{\circ} \mathrm{C}\right)$, con un mayor rango de temperaturas $\left[11,5^{\circ} \mathrm{C}-5,5^{\circ} \mathrm{C}\right]$, eso indicó que las temperaturas sufrieron fuertes descensos, por lo que las heladas fueron de fuerte intensidad y abarcaron un amplio período de ocurrencias, limitando la actividad agrícola solo a pocos cultivos. 
La caracterización de las heladas, puso en evidencia que la alfalfa, sembrada en casi toda la región Puno, está a expuesta durante gran parte del año a las heladas, tanto meteorológicas como agronómicas, lo cual tiene un fuerte impacto sobre la producción de la biomasa aérea y materia seca. De esta manera, en las partes más altas de la región, donde la temperatura del aire desciende a $0{ }^{\circ} \mathrm{C}$ o a una temperatura baja aún más crítica, las heladas están presentes todos los días del año, significando que el período libre de heladas fue de 0 días. Los valores de temperaturas en los 3 clústeres estuvieron por debajo de aquellos sugeridos para el crecimiento de la alfalfa por Del Pozo, 1971, D’Attlellis, 2005; Mayhua et al., 2008. Sin embargo, el amortiguamiento ocasionado por la presencia del Lago Titicaca, tiene un rol fundamental en el crecimiento de la alfalfa y otros cultivos y producción.

\section{Conclusiones}

Las heladas meteorológicas fueron y son un peligro persistente durante todos los años en la región Puno, en todos los casos, la frecuencia anual de heladas fue del 100 $\%$. Estacionalmente, la mayor frecuencia (22\%) de heladas fue registrada entre los meses de mayo a agosto y en el período setiembre a abril, lapsos en los cuales se desarrolla la actividad agrícola en la región. En el período diciembrefebrero, en el cual abarca la duración de la etapa fenológica más crítica, la frecuencia de heladas fue del 16\%.

Las primeras heladas, ocurren entre el 28 de enero y 14 de abril y su intensidad media oscila entre $-3,1{ }^{\circ} \mathrm{C}$ (Taraco) y $-0,3{ }^{\circ} \mathrm{C}$ (Juliaca). La última helada meteorológica, ocurre entre el $1^{\circ}$ de noviembre y el 26 de diciembre y la intensidad media oscila entre $-2,6^{\circ} \mathrm{C}$ (Taraco) y $-0,4$ ${ }^{\circ} \mathrm{C}$ (Arapa). El período libre de heladas meteorológicas fluctúa entre 160 y 200 días para las estaciones agrupadas en el Clúster 1, entre 120 y 150 días para la estaciones del Clúster 2 y 50 días en promedio para la estación que pertenece al Clúster 3.

El modelo matemático resultante estableció la estrecha relación lineal que existe entre la variable temperatura y el rendimiento, impactando de manera significativa en la producción de alfalfa. Se demostró que si la temperatura promedio anual aumenta en $1{ }^{\circ} \mathrm{C}$, los rendimientos para el cultivo de alfalfa (Medicago sativa $\mathrm{L}$.) se incrementan en $838 \mathrm{~kg}$ por hectárea.

\section{Literatura citada}

Alarcón, C. 1991. Caracterización agroclimática del Departamento de Cuzco. Tesis Ing. Meteorólogo. Universidad Nacional Agraria La Molina. Lima, Perú.

Alexander M. 1994. Introducción a la microbiología del suelo. Editor S.A. México DF.

Anaya, M. 2004. Cultivo de alfalfa con riego por goteo superficial. México DF Banco Central de Reserva. Síntesis Económica de Puno, Lima.

Collantes, R. 2007. Comparativo del rendimiento y calidad de cultivares mejoradas de cebada (Hordeum vulgare) en dos localidades de la sierra central del Perú. Tesis Ing. Agrónomo. Universidad Nacional Agraria la Molina. Lima, Perú.

Dallas, E. 1998. Métodos multivariados aplicados al análisis de datos. Brooks Cole Publishing Company. México DF.

D’Attlellis, 2005. Alfalfa (Medicago sativa) producción de semilla. Dirección Provincial de Programación del Desarrollo. Ministerio de la Producción de la provincia de Catamarca. Cajamarca.

De Fina, A. y Ravello, C. 1979. Climatología y fenología agrícolas. Editorial Universitaria de Buenos Aires, tercera edición. Buenos Aires, Argentina.

Del Pozo, M. 1974. La alfalfa, su cultivo y aprovechamiento. Edit. Mundi - Prensa. Madrid, España.

Domenech, J. 1985. Métodos estadísticos: modelo lineal de regresión. Barcelona, España.

Elías, F. y Castellvi, F. 2001. Agrometeorología. Ediciones Mundi-Prensa, segunda edición, Madrid

Etxeberria, J. 1999. Regresión Múltiple. Cuadernos de Estadística. Ed. La Muralla S.A. Hespérides, Salamanca.

Gómez, F. 2005. Praticultura. Ed. Universidad Politécnica de Valencia. Valencia, España.

Lasso, L. 1997. Anotaciones sobre el fenómeno de las heladas. Instituto Colombiano de Hidrología, Meteorología y Adecuación de Tierras-HIMAT. Segunda Edición. Bogotá, Colombia.

Manarelli, L. 1988. Temperatura mínima. En: Evaluación agroclimática del departamento de Puno. Dirección General de Agrometeorología. SENAMHI, Lima.

Martínez, L.; Ibacache, A. y Rojas, L. 2007. Efecto de las heladas en la agricultura. Boletín INIA Nº165. INIA Intihuasi. Coquimbo, Santiago de Chile.

Matías, L.; Fuentes, A. y García, F. 2007. Heladas (pp. 3254). Disponible en: http://www.cenapred.unam.mx/es/ publicaciones/archivos/1412008fasc._Heladas_2007. pdf.

Mayhua, P.; Quispe, E.; Contreras, J.; Ramos, Y.; Guillén, H. 2008. Instalación y conservación de pastos cultivados en altura. INCAGRO. Huancavelica, Perú.

OMS [Organización Meteorológica Mundial]. 1996. Guía de instrumentos y métodos de observación meteorológicos. Sexta edición, OMM No8. Ginebra, Suiza.

SENAMHI [Servicio Nacional de Meteorología e Hidrología]. 2010. Atlas de heladas del Perú. Lima, Perú.

SENAMHI [Servicio Nacional de Meteorología e Hidrología]. 2012. Caracterización agroclimática de la Región Apurímac. Lima, Perú.

Soil Survey Staff. 2014. Departamento de Agricultura de los Estados Unidos. Servicio de Conservación de Recursos Naturales. Décima segunda Edición. 